\title{
Identification of blood protein biomarkers that aid in the clinical assessment of patients with malignant glioma
}

\author{
BAOGANG J. XU ${ }^{1,2,3,8}$, QI A. AN ${ }^{4}$, SUPRIYA SRINIVASA GOWDA ${ }^{1}$, WENWEI YAN ${ }^{3}$, \\ LARRY A. PIERCE ${ }^{1}$, TY W. ABEL ${ }^{2,5}$, SARAH Z. RUSH ${ }^{6}$, MICHAEL K. COOPER ${ }^{2,7}$, \\ FEI YE ${ }^{4}$, YU SHYR ${ }^{2,4}$, KYLE D. WEAVER ${ }^{1,2}$ and REID C. THOMPSON ${ }^{1,2}$ \\ ${ }^{1}$ Department of Neurological Surgery, Vanderbilt University Medical Center; ${ }^{2}$ Vanderbilt-Ingram Cancer Center; \\ Departments of ${ }^{3}$ Cancer Biology, ${ }^{4}$ Biostatistics, ${ }^{5}$ Pathology, ${ }^{6}$ Pediatrics, and ${ }^{7}$ Neurology, Vanderbilt University \\ Medical Center, and Veterans Affairs Tennessee Valley Healthcare System, Nashville, TN, USA
}

Received November 5, 2011; Accepted December 22, 2011

DOI: $10.3892 /$ ijo.2012.1355

\begin{abstract}
Analyzing molecular biomarkers using blood is an important approach for clinical assessment of malignant glioma. We investigated a molecular proteomic biomarkerbased approach for glioblastoma using patients' blood samples. The expression levels of a list of candidate proteins were quantified in plasma and serum samples from two different cohorts of patients with malignant glioma and normal controls. The biological function was studied for one of the identified markers. Additionally, the prognostic significance of protein marker expression was measured by survival analysis. As a result, protein biomarkers associated with malignant glioma were identified from the blood specimens and five of the protein biomarkers were common to both cohorts. Immunohistochemical analysis demonstrated that many of the protein biomarkers identified in peripheral blood specimens were expressed in malignant gliomas. Staining levels for one of the biomarkers, MIP-1 $\alpha$, was found to correlate with WHO grade among invasive gliomas, and we demonstrate that MIP- $1 \alpha$ promotes human glioblastoma cell proliferation and migration. Additionally, four prognostic protein biomarkers were identified. In conclusion, we demonstrate that both peripheral blood plasma and serum specimens are highly valuable and complementary to each other in the quest for protein biomarkers of malignant glioma. Sets of novel protein biomarkers were identified that may aid in the diagnosis and prognosis of patients with malignant glioma.
\end{abstract}

Correspondence to: Dr Baogang J. Xu, ${ }^{8}$ Present address: University of Tennessee Health Science Center, School of Medicine, Memphis, TN 38163, USA

E-mail: bxu5@uthsc.edu

Key words: malignant glioma, proteomics, biomarkers, serum, plasma, prognosis

\section{Introduction}

Gliomas are the most common tumors of the central nervous system in adults. Malignant glioma, or high-grade glioma, refers to World Health Organization (WHO) grades III and IV glioma. Malignant glioma carries a significantly lower survival rate compared with low-grade, or WHO grades I and II glioma. A reliable, noninvasive, and convenient method for deciphering whether a glioma is malignant or monitoring response to therapies would be highly beneficial. Molecularbased biomarkers will continue to play important roles in assisting glioma diagnosis, prognosis, and therapy. Analyzing molecular biomarkers in blood is an attractive approach for clinical assessment of malignant glioma. Blood can be sampled frequently with minimal risk, and detecting malignant glioma associated proteins in peripheral blood is possible because brain tumor vasculature is known to be leaky compared with healthy blood-brain barrier (1).

Various studies suggest that proteins associated with malignant glioma can be detected using blood samples. For example, serum-borne epidermal growth factor receptor (EGFR) levels were shown to be significantly different when comparing healthy controls with malignant glioma patients and EGFR serum levels are associated with prognosis (2). Levels of a proliferation-inducing ligand (APRIL) have been found to be elevated in the sera of glioblastoma (GBM) patients when compared to non-tumor controls (3). Another study suggests serum levels of glial fibrillary acidic protein (GFAP) are correlated with tumor volume in high-grade gliomas (4). Recently, insulin-like growth factor binding protein-2 (IGFBF-2), measured in plasma, was shown to be up-regulated in high-grade glioma patients when compared with grade II glioma patients and normal controls, and its expression levels can predict clinical outcomes of patients with highgrade gliomas (5). These studies demonstrated the promise of studying blood-based protein biomarkers for malignant glioma; however, only a single protein candidate was tested in each study. With the advent of proteomic technologies, a high-throughput and multiplex approach holds the promise of 
identifying additional, including previously unknown, protein biomarkers for malignant glioma.

Furthermore, previous studies have not addressed whether serum or plasma samples should be the preferred blood samples. Plasma is the liquid component of the unclotted whole blood after blood cells are removed. In contrast, serum is the top liquid component after the whole blood is clotted and centrifuged. A portion of the protein content in blood is removed in serum in the form of fibrin clot. Considering the two qualitatively different types of blood samples, a study should be performed to evaluate the potential differences in protein biomarker expressions in both serum and plasma samples from malignant glioma patients.

The goal of this study is to identify and compare both serum and plasma protein biomarker candidates that may aid in the clinical assessment of malignant glioma. We first aimed to examine a list of proteins in both plasma and serum samples obtained from malignant glioma patients and healthy controls. The differentially expressed protein biomarker candidates were further subjected to immunohistochemical analysis to assess their expressions in glioma tissues. The biological functions for a selected protein biomarker related to malignant glioma progression were explored. The protein biomarker candidates that were commonly identified in both serum and plasma samples were selected and compared. Finally, the protein biomarker candidates with potential diagnostic values were analyzed.

\section{Materials and methods}

Study population and multiplex immunoassays. Immediately before tumor resection surgery at Vanderbilt University Medical Center (VUMC), EDTA-plasma samples were collected from 28 consenting malignant glioma patients. These include 23 cases of World Health Organization (WHO) grade IV GBM and 5 cases of WHO grade III glioma. The age distribution for the 28 patients was $56 \pm 19$ (average \pm SD) and included seven females and 22 males. Serum samples from 20 GBM patients who underwent tumor resection surgery were collected by the Brain Tumor Foundation of Canada (BTFC). These patients had an age distribution of $60 \pm 13$ (average \pm SD) and included eight females and 12 males. The samples were kept frozen until analysis. After the blood samples were collected and processed, frozen plasma or serum samples were analyzed by Rules-Based Medicine (RBM, Austin, TX), a laboratory certified by Clinical Laboratory Improvement Amendments (CLIA), using a Luminex multiplex immunoassay. A total of 367 normal plasma samples and 1278 normal serum samples previously analyzed by RBM were used as controls. The donors for control serum samples had an age distribution of $52 \pm 12$ (average \pm SD) and included 614 females and 664 males. The studies were approved by local institutional review boards, and patients' informed consents were obtained for the collection of the plasma or serum samples.

The blood samples were processed and analyzed using a robotics-based automated instrument, which performed sample dilution, addition of antibodies, incubation, and sample transfer steps. As previously described (6), each analyte was quantified based on an eight-point standard curve, and the test performance was confirmed using quality control samples at low, medium, and high levels for each analyte in duplicate. The standard and quality control samples were mixed in a plasma or serum matrix to match the sample background. The assays were absolute quantitative, and the results obtained for samples were compared with established standard values. The least detectable level was determined as the mean plus 3 standard deviations as derived from 20 blank readings.

Immunohistochemistry and quantification. Paraffin-embedded formalin-fixed human GBM tissue slides were warmed on a slide warmer at $58^{\circ} \mathrm{C}$ overnight, followed by deparaffinization and rehydration with xylene and ethanol, respectively. Tissue microarray slides containing different grades of human glioma cases obtained from AccuMax Array (BioCarta, San Diego, CA) or Vanderbilt University (7) were similarly processed. For antigen retrieval, the slides were treated with $10 \mathrm{mM}$ citrate buffer ( $\mathrm{pH} \mathrm{6.0)}$ at $95^{\circ} \mathrm{C}$ for $25 \mathrm{~min}$, and the slide for stem cell factor was treated with pepsin at $37^{\circ} \mathrm{C}$ for $15 \mathrm{~min}$. The slides were quenched with $3 \%$ hydrogen peroxide and blocked with serumfree blocking protein (DakoCytomation, Inc, Carpinteria, CA) for $30 \mathrm{~min}$. The slides were incubated with antibodies specific for Stem Cell Factor (1:50, cat. no. sc-13126, Santa Cruz, CA), Endothelin-1 (1:50, cat. no. ab2786, Abcam, Cambridge, MA), TIMP-1 (1:25, cat. no. M7293, Dako, Denmark), or MIP-1 $\alpha$ (1:25, cat. no. ab32609, Abcam) overnight at $4^{\circ} \mathrm{C}$. In addition, human GBM frozen tissue sections were prepared and warmed on a slide warmer at $37^{\circ} \mathrm{C}$ for $5 \mathrm{~min}$. Tissue sections were fixed with $4 \%$ paraformaldehyde at room temperature for $15 \mathrm{~min}$ and quenched with $3 \%$ hydrogen peroxide. After blocking with serum-free blocking protein for $30 \mathrm{~min}$, each slide was incubated with antibodies specific for CA19-9 (1:50, cat. no. TA888, Zymed, Carlsbad, CA) or MMP2 (1:40, cat. no. HPA001939, Sigma, St. Louis, MO) overnight at $4^{\circ} \mathrm{C}$. All of the slides were incubated with a biotinylated secondary antibody (Biotinylated Link Universal, Dako) for $30 \mathrm{~min}$, and subsequently reacted with a streptavidin-peroxidase conjugate (Dako) and 3-amino9-ethylcarbazole (Vector Laboratories, Burlingame, CA), respectively. The slides were counterstained with hematoxylin and cover-slipped. Positive controls were performed according to the manufacturers' suggestions.

Quantitative analysis of MIP-1 $\alpha$ staining in the tissue microarray (TMA) was performed similar to a procedure previously described (8). Briefly, a system composed of an Olympus BX40 microscope (Olympus American, Miami, FL), a Retiga FAST 1394 color digital camera (QImaging, British Columbia, Canada), and Bioquant Nova Prime imaging analysis software (Bioquant, Nashville, TN) was used. Under a constant state of exposure control and a consistent intensity setting, images were captured for the tissue cores, and threshold settings were applied to measure percentage of positively stained areas. Manual editing of fields was used to eliminate nonspecific artifacts.

Glioblastoma cell proliferation and migration assays. Human glioblastoma T98G cells were incubated at $37^{\circ} \mathrm{C}$ and $5 \% \mathrm{CO}_{2}$ with Eagle's minimum essential medium (EMEM) supplemented with $10 \%$ FBS. The cells were plated in 96-well plates. After reaching $80 \%$ confluency, the cells were washed with PBS and replaced with serum-free EMEM containing recombinant MIP-1 $\alpha$ (Invitrogen, Carlsbad, CA) at concentrations of $0,0.001,0.01,0.1,1,10$, and $100 \mathrm{ng} / \mathrm{ml}$. The cells were 


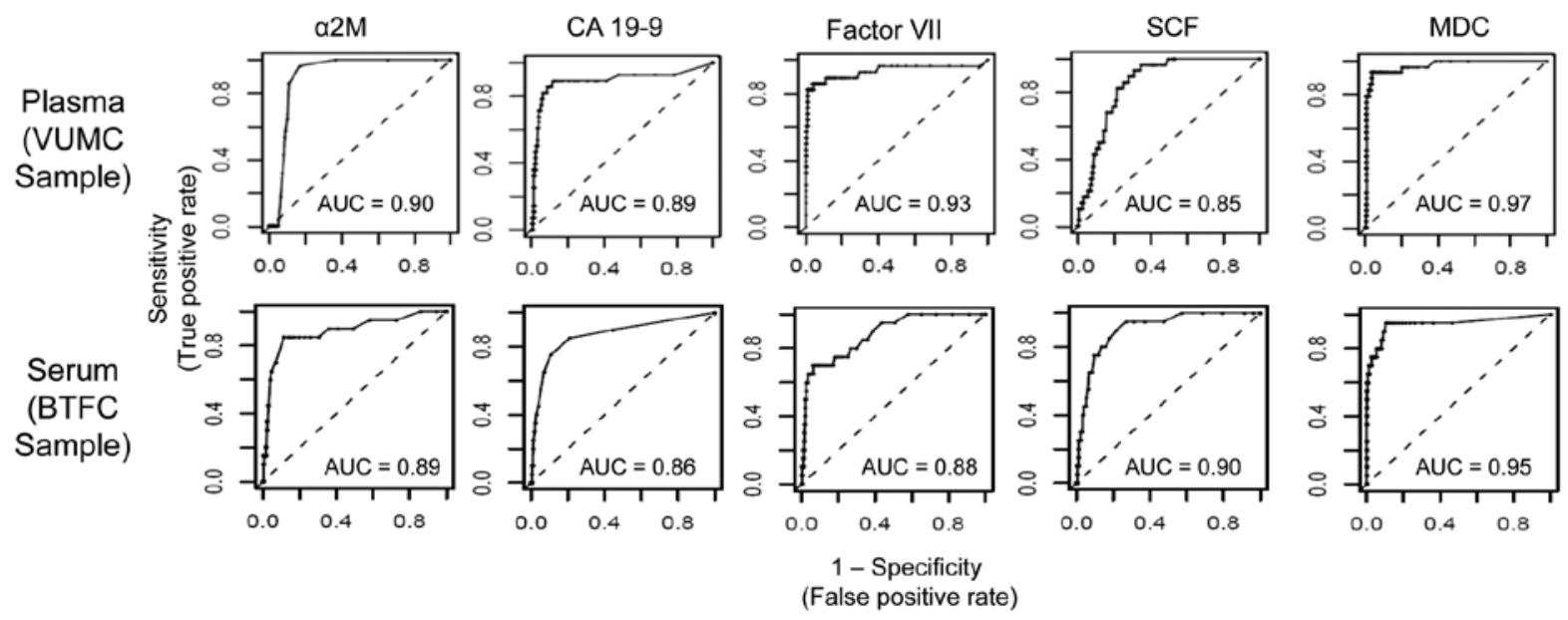

Figure 1. Five blood-based protein biomarkers show consistent classification accuracies of high-grade glioma patients and controls in the two independent sample cohorts.

incubated for $48 \mathrm{~h}$ and WST-1 proliferation assays (Roche, Indianapolis, IN) were performed according to manufacturer instructions. T98G cell migration assays were performed in 6-well plates. After the cells reached $100 \%$ confluency, scratch-wounds were created. The cells were washed and incubated with serum-free EMEM with MIP- $1 \alpha$ at the following concentrations: $0.01,0.1,1,10$, and $100 \mathrm{ng} / \mathrm{ml}$. Wound closure was measured at $0,8,20,30$ and $48 \mathrm{~h}$ time points. The images of the percentage of wound closures were calculated at each time point.

Statistical analysis. Univariate logistic regression models were fitted individually for each protein to discriminate high-grade glioma patients from normal controls. Based on logistic regression estimates, area under the curve (AUC) based on receiver operating characteristic (ROC) analyses was analyzed for optimal sensitivity and specificity. The Wilcoxon rank sum test was performed to assess the differences in protein expression levels between high-grade glioma patients and normal control patients. Kaplan-Meier survival analyses were performed for patients who underwent tumor resection surgeries at the Vanderbilt University Medical Center. Significance of differences in survival between patients with a protein level above median and those below median were calculated using the log-rank test. Proteins with $>20 \%$ of their expression levels below the least detectable level were excluded as potential biomarker candidates. Statistical analysis was performed using the free, open-source R statistical software version 2.6.2 (www.r-project.org).

The Spearman's rank correlation test was performed to determine the significance of differences in the WST-1 cell proliferation assay and cell wound closure migration assay. Three replicate experiments were performed for each assay.

\section{Results}

Blood-based protein biomarker for classification of high-grade glioma from controls. The expression of 75 proteins (Table I) in peripheral blood specimens were analyzed in our study based upon the availability of Luminex-based multiplex assays. Plasma and serum samples are widely used for clinical purposes and it is not clear which type of blood specimen is better suited for protein biomarker discovery for malignant glioma. Therefore, both types of peripheral blood specimens were analyzed in the study. Plasma specimens were obtained from the 28 patients with malignant glioma and from 367 healthy controls, and serum specimens were obtained from an additional 20 patients with malignant glioma and an additional 1278 healthy controls.

To identify peripheral blood protein markers that might identify patients with malignant glioma, receiver operating characteristic (ROC) curves were analyzed. An area under the ROC curve (AUC) of $>85 \%$ was utilized to identify 16 proteins from plasma specimens (Table II) and 9 proteins from serum specimens (Table III) whose expression levels might differentiate patients with malignant glioma from controls. Five of the proteins, $\alpha$-2-macroglobulin (A2M), cancer antigen 19-9 (CA 19-9), factor VII, Macrophage-derived Chemokine (MDC), and stem cell factor (SCF), were identified as markers of patients with malignant glioma in both plasma and serum specimens. Significant differences in the protein expression levels between the malignant glioma patient group and control group were also found using Wilcoxon tests $(\mathrm{p}<0.0001)$. By this method, unique and complementary protein biomarkers were selected using plasma and serum samples.

Consistent protein biomarkers from the two independent cohorts. Plasma and serum specimens represent two distinct types of blood samples that are commonly used for protein biomarker analysis. The analysis of plasma and serum specimens derived from two independent cohorts of patients may provide the opportunity to validate protein biomarkers and to compare the analysis of distinct sample types. Comparison of the ROC curves for the five protein biomarkers identified in both plasma and serum specimens, A2M, CA 19-9, factor VII, MDC, and $\mathrm{SCF}$, indicates similar AUC measurements and classification accuracies for distinguishing patients with malignant glioma from controls in the two cohorts (Fig. 1). Likewise, comparison of box-and-whisker plots for analyte measurements in the two cohorts of patient and control specimens revealed consistent distributions and median levels. Specifically, comparing 
Table I. The 75 proteins used in the proteomics analysis.

\begin{tabular}{|c|c|c|c|}
\hline No. & Protein & No. & Protein \\
\hline 1 & $\alpha-1$-antitrypsin & 39 & Interleukin-16 \\
\hline 2 & Adiponectin & 40 & Interleukin-18 \\
\hline 3 & $\alpha$-2-macroglobulin & 41 & Interleukin- $1 \alpha$ \\
\hline 4 & $\alpha$ fetoprotein & 42 & Interleukin-1 $\beta$ \\
\hline 5 & Apolipoprotein A1 & 43 & Interleukin-3 \\
\hline 6 & Apolipoprotein CIII & 44 & Interleukin-5 \\
\hline 7 & Apolipoprotein $\mathrm{H}$ & 45 & Interleukin-6 \\
\hline 8 & $\beta$-2-microglobulin & 46 & Interleukin-7 \\
\hline 9 & Brain-derived neurotrophic factor & 47 & Interleukin-8 \\
\hline 10 & Complement 3 & 48 & Insulin \\
\hline 11 & Cancer antigen 125 & 49 & Leptin \\
\hline 12 & Cancer antigen 19-9 & 50 & Lipoprotein \\
\hline 13 & Calcitonin & 51 & Lymphotactin \\
\hline 14 & Carcinoembryonic antigen & 52 & Monocyte Chemotactic Protein-1 \\
\hline 15 & Creatine kinase MB & 53 & Macrophage-Derived Chemokine \\
\hline 16 & C-Reactive protein & 54 & Macrophage Inflammatory Protein $1 \alpha$ \\
\hline 17 & Epidermal growth factor & 55 & Macrophage Inflammatory Protein $1 \beta$ \\
\hline 18 & Epithelial neutrophil activating peptide 78 & 56 & Matrix Metalloproteinase 2 \\
\hline 19 & Endothelin 1 & 57 & Matrix metalloproteinase 3 \\
\hline 20 & Eotaxin & 58 & Matrix metalloproteinase 9 \\
\hline 21 & Erythropoietin & 59 & Myoglobin \\
\hline 22 & Fatty acid binding protein & 60 & Plasminogen activator inhibitor type I \\
\hline 23 & Factor VII & 61 & Prostatic acid phosphatase \\
\hline 24 & Ferritin & 62 & Prostate-specific antigen, free \\
\hline 25 & Fibroblast growth factor-basic form & 63 & Regulated upon activation, normal T-cell expressed and secreted \\
\hline 26 & Fibrinogen & 64 & Serum amyloid $\mathrm{P}$ \\
\hline 27 & Growth hormone & 65 & Stem cell factor \\
\hline 28 & Granulocyte macrophage colony stimulating factor & 66 & Thyroxine binding globulin \\
\hline 29 & Glutathione S-transferase & 67 & Tissue factor \\
\hline 30 & Intercellular adhesion molecule 1 & 68 & Tissue inhibitor of metalloproteinase 1 \\
\hline 31 & Immunoglobulin A & 69 & Tumor necrosis factor- $\alpha$ \\
\hline 32 & Immunoglobulin $\mathrm{E}$ & 70 & Tumor necrosis factor- $\beta$ \\
\hline 33 & Immunoglobulin M & 71 & Thrombopoietin \\
\hline 34 & Interleukin-10 & 72 & Thyroid stimulating hormone \\
\hline 35 & Interleukin-12p40 & 73 & Vascular cell adhesion molecule 1 \\
\hline 36 & Interleukin-12p70 & 74 & Vascular endothelial growth factor \\
\hline 37 & Interleukin-13 & 75 & von Willebrand factor \\
\hline 38 & Interleukin-15 & & \\
\hline
\end{tabular}

analyte measurements in patients with high-grade glioma to controls in plasma and serum specimens, respectively; A2M was increased 2.17- and 2.19-fold, CA 19-9 was increased 11.28- and 5.00-fold, factor VII was increased 2.43- and 2.10-fold, SCF was increased 2.78- and 2.06-fold, and MDC was decreased 6.3- and 5.0-fold (Fig. 2). Therefore, A2M, CA 19-9, factor VII, MDC, and SCF represent protein biomarkers that may be used to identify patients with malignant glioma in independent cohorts with similar accuracy in either peripheral blood plasma or serum specimens.
Expression of the proteomic biomarkers in malignant glioma tissues. Exploration of the source of the proteins that were identified in the blood samples will increase our understanding of the protein biomarker candidates. Multiple cell types associated with malignant glioma development, such as tumor parenchyma cells, endothelial cells, or tumor associated lymphocytes, may be contributing the proteins. To study the source of the protein biomarkers identified in peripheral blood specimens in malignant gliomas, immunohistochemistry (IHC) was performed based on the availability of suitable antibodies. The following 
Table II. The sensitivity and specificity for the potential plasma protein biomarkers in classification of high-grade glioma patients and controls.

\begin{tabular}{lccc}
\hline Protein name & Sensitivity & Specificity & p-value \\
\hline$\alpha$-2-macroglobulin & 0.96 & 0.83 & $4.9 \mathrm{E}-13$ \\
Apolipoprotein A1 & 0.79 & 0.86 & $1.9 \mathrm{E}-12$ \\
$\beta$-2-microglobulin & 0.79 & 0.92 & $3.9 \mathrm{E}-11$ \\
Cancer antigen 19-9 & 0.86 & 0.91 & $7.9 \mathrm{E}-12$ \\
Complement 3 & 0.75 & 0.88 & $3.9 \mathrm{E}-11$ \\
Endothelin-1 & 0.96 & 0.84 & $<1.0 \mathrm{E}-16$ \\
Factor VII & 0.86 & 0.96 & $2.3 \mathrm{E}-14$ \\
Fibrinogen & 0.71 & 0.91 & $6.5 \mathrm{E}-11$ \\
IgA & 0.96 & 0.82 & $5.3 \mathrm{E}-14$ \\
IL 16 & 0.75 & 0.89 & $1.4 \mathrm{E}-11$ \\
MDC & 0.93 & 0.97 & $7.8 \mathrm{E}-17$ \\
MIP-1 $\alpha$ & 1.00 & 0.88 & $1.8 \mathrm{E}-15$ \\
MMP-2 & 0.96 & 0.84 & $1.1 \mathrm{E}-13$ \\
Stem cell factor & 0.93 & 0.69 & $4.4 \mathrm{E}-11$ \\
Thyroxine binding globulin & 0.79 & 0.93 & $4.0 \mathrm{E}-15$ \\
\hline
\end{tabular}

The p-values associated with the protein expression level differences between the malignant glioma patient group and control group are listed.

proteins showed positive staining in human GBM tissues, CA 19-9, MMP-2, TIMP-1, Endothelin-1, SCF and MIP-1 $\alpha$ (Fig. 3). MMP2, CA 19-9, and endothelin-1 staining appear to be limited to the cytoplasm of tumor cells. TIMP-1 staining appears to be limited to an area of vascular proliferation but not to the tumor cells. SCF and MIP-1 $\alpha$ staining was also detected in blood vessels. IL-10 and IL-16 IHC expression in malignant glioma
Table III. The sensitivity and specificity for the potential serum protein biomarkers in classification of high-grade glioma patients and controls.

\begin{tabular}{lccc}
\hline Protein name & Sensitivity & Specificity & p-value \\
\hline$\alpha$-2-macroglobulin & 0.85 & 0.89 & $1.5 \mathrm{E}-11$ \\
Apolipoprotein C-III & 0.70 & 0.95 & $7.6 \mathrm{E}-7$ \\
Brain derived & 0.75 & 0.97 & $3.3 \mathrm{E}-12$ \\
neurotrophic factor & & & \\
Cancer antigen 19-9 & 0.75 & 0.90 & $8.6 \mathrm{E}-4$ \\
Factor VII & 0.70 & 0.94 & $5.8 \mathrm{E}-8$ \\
IL 10 & 0.95 & 0.85 & $3.1 \mathrm{E}-10$ \\
MDC & 0.95 & 0.90 & $1.5 \mathrm{E}-12$ \\
MMP-3 & 0.75 & 0.87 & $2.0 \mathrm{E}-8$ \\
Stem cell factor & 0.90 & 0.78 & $1.1 \mathrm{E}-3$ \\
\hline
\end{tabular}

The p-values associated with the protein expression level differences between the malignant glioma patient group and control group were listed.

tumor cells is demonstrated in the Human Protein Atlas (www. proteinatlas.org) (9). The staining patterns of multiple protein biomarkers, such as TIMP-1, SCF and MIP-1 $\alpha$, shown in our patients indicate their high expressions in tumor and endothelial cells.

MIP-1 $\alpha$ expression and effects on glioma cell proliferation and migration. ROC curve analysis indicated that MIP- $1 \alpha$ in the peripheral blood is one of the most sensitive indicators of malignant glioma (Table II). MIP- $1 \alpha$ is a member of the CC

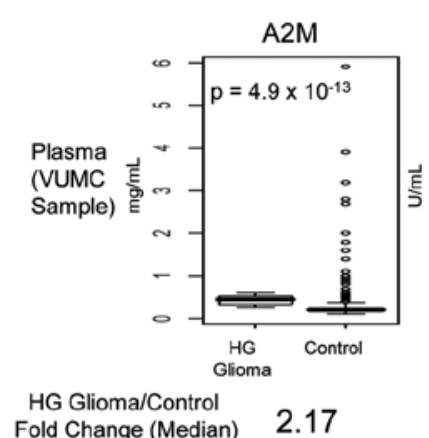

Fold Change (Median) 2.17
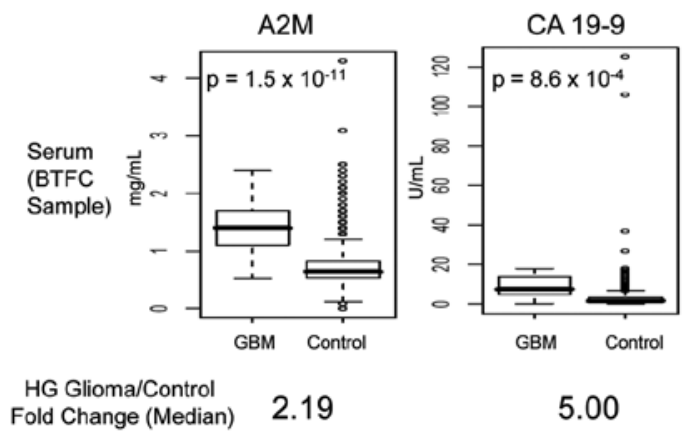

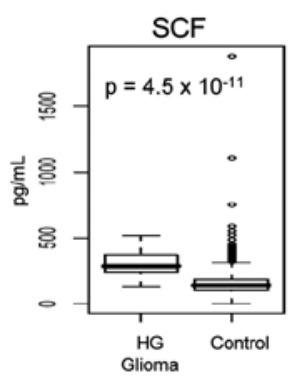

2.78

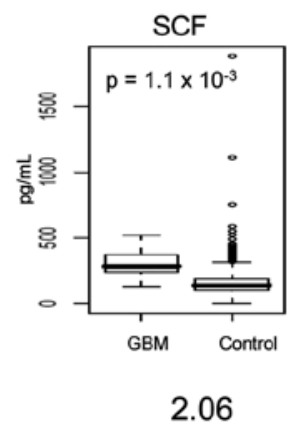

Figure 2. Five blood-based protein biomarkers show consistent fold-changes among high-grade glioma patients and controls in the samples from the two independent cohorts. 


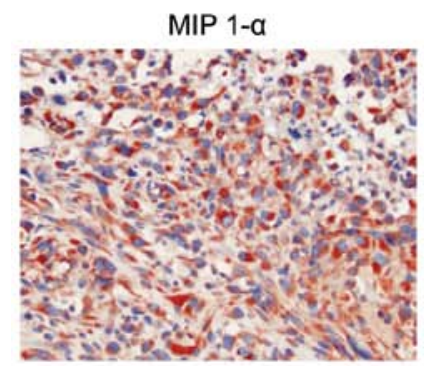

CA 19-9

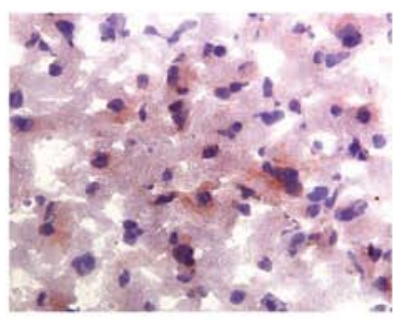

MMP-2

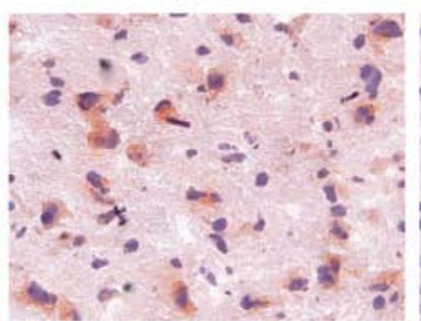

Endothelin-1

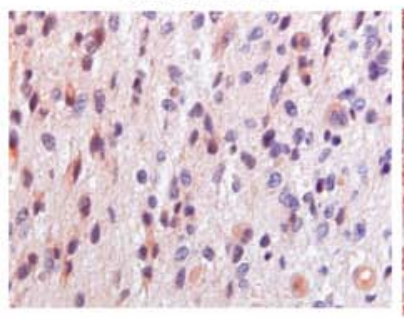

TIMP -1

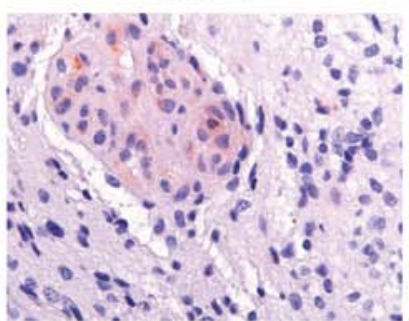

SCF

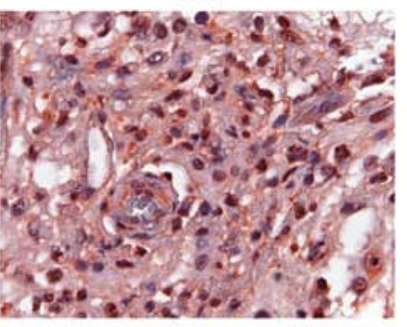

IL-10 *



IL-16 *

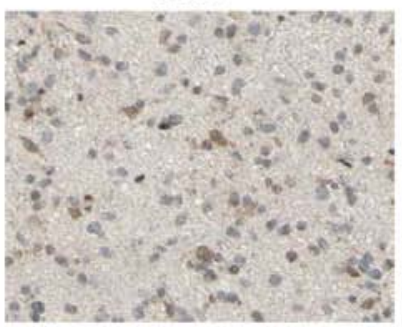

Figure 3. Immunohistochemical staining shows positive expression of different blood-based protein biomarker candidates in human glioblastoma tissues. "IL-16 and IL-10 IHC staining in human high-grade glioma tissues are shown in the Human Protein Atlas website (www.proteinatlas.org).
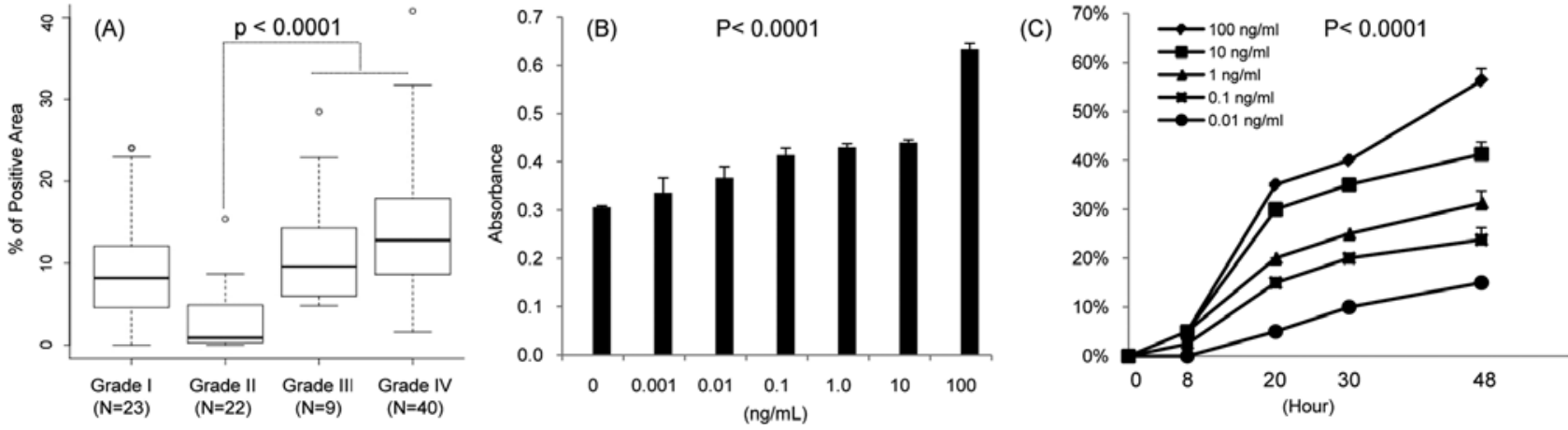

Figure 4. MIP-1 $\alpha$ expression and function in human gliomas. (A) Quantification of MIP-1 $\alpha$ percentage of positive staining area in glioma TMA that contained grade I $(\mathrm{N}=23)$, grade II $(\mathrm{N}=22)$, grade III $(\mathrm{N}=9)$, and grade IV $(\mathrm{N}=40)$ glioma cases. (B) MIP-1 $\alpha$ stimulates human T98G glioblastoma cells proliferation using WST-1 assay. Different dosage of MIP-1 $\alpha$ treatment was found to be positively correlated with increased cell proliferation (p<0.0001, error bar, SD). (C) MIP-1 $\alpha$ stimulates human T98G glioblastoma cells migration using wound closure assay. Positive correlation was found between increasing dosage of MIP-1 $\alpha$ treatment and the increased rate of cell migration $(\mathrm{p}<0.0001$, error bar, $\mathrm{SD})$.

chemokine family, whose expression and function in GBM are not well understood (10). However, in patients with multiple myeloma, MIP- $1 \alpha$ is produced by myeloma cells and protein levels in the bone marrow plasma correlate with disease stage (11). Furthermore, MIP- $1 \alpha$ is a potent growth, survival and chemotactic factor for myeloma cells (12).

To determine whether MIP-1 $\alpha$ expression levels correlate with tumor grade, MIP-1 $\alpha$ IHC was performed on tissue microarrays (TMA) of WHO grades I-IV gliomas. The percentage of positive staining area for MIP- $1 \alpha$ for each tissue core was quantified using Bioquant imaging analysis software. The only significant difference for MIP-1 $\alpha$ is between grade II and other glioma grades (Fig. 4A). A significant increase was observed when comparing the grade II cases versus combined grade III and grade IV cases. Interestingly, grade I cases was also found to have a higher expression of MIP-1 $\alpha$ comparing with the grade II cases.

T98G human glioblastoma cell proliferation response to MIP-1 $\alpha$ with increasing dosage treatment $(0.01,0.1,1.0,10$ and $100 \mathrm{ng} / \mathrm{ml}$ ) was measured using WST-1 cell proliferation assay. Different dosage of MIP- $1 \alpha$ treatment was found to be positively correlated with increased cell proliferation $(\mathrm{p}<0.0001)$ (Fig. 4B). In addition, T98G cell migration response to MIP-1 $\alpha$ with increasing dosage $(0.1,1.0,10$, and $100 \mathrm{ng} / \mathrm{ml})$ treatment was assayed using wound-closure cell migration assay. Positive correlation was found between increasing dosage of MIP-1 $\alpha$ treatment and the increased rate of cell migration $(\mathrm{p}<0.0001)$ (Fig. 4C).

Survival analysis. In addition to the use of biomarkers to identify patients with malignant glioma, biomarkers associated with survival may also serve important roles in clinical assessment. We analyzed the prognostic significance for all of the 75 proteins (Table I) that were measured in the plasma specimens utilized in our study. Median protein expression level was used as the cutoff point to divide the patients into two subgroups. Of the 75 analytes, four proteins (growth hormone, IgE, IL-16, and VEGF) were found to have significant prognostic value based 

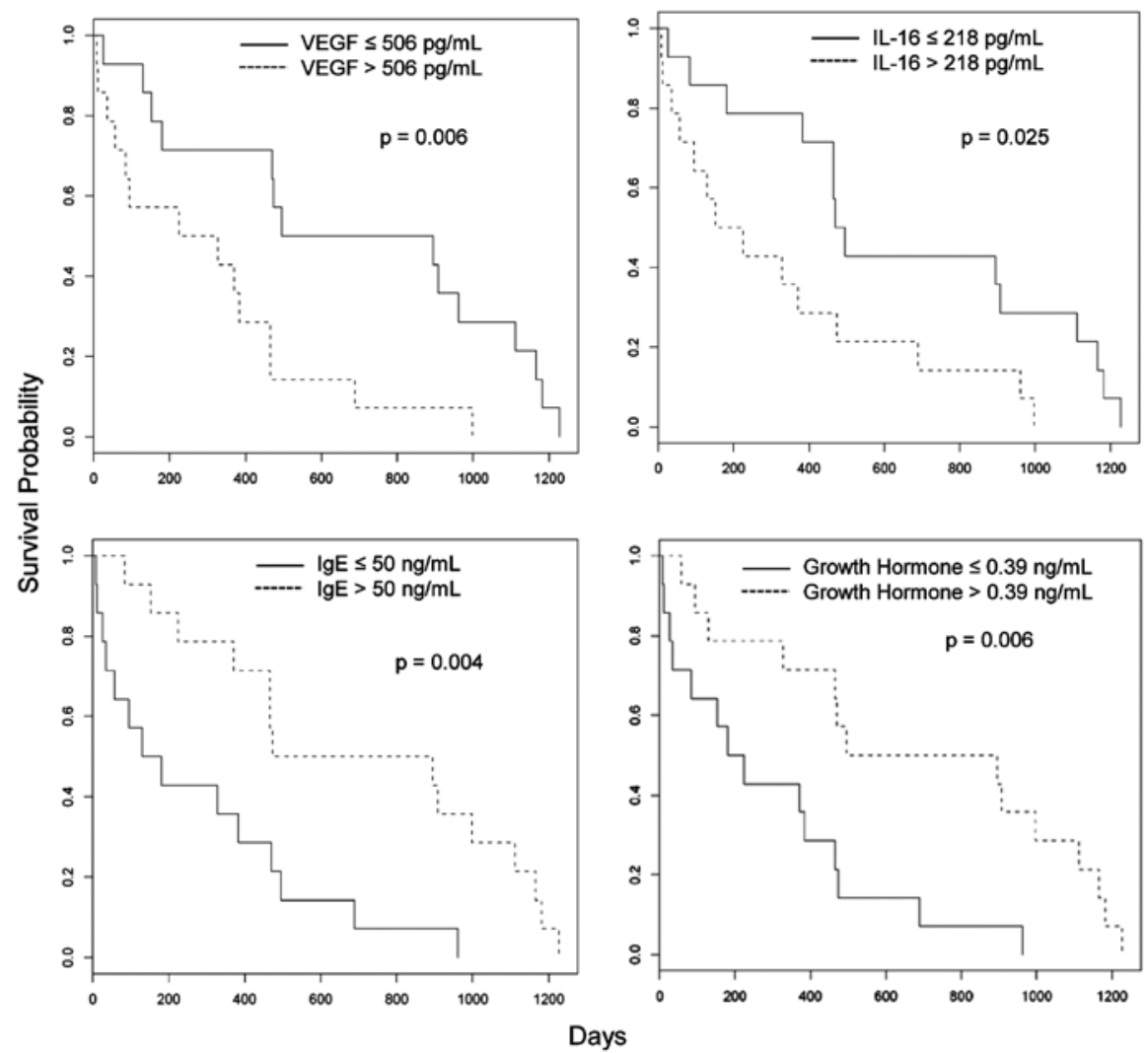

Figure 5. Kaplan-Meier survival plots show that four plasma protein biomarkers (VEGF, IL-16, IgE, and GH) are associated with prognosis in high-grade glioma patients.

on the log-rank test (Fig. 5). The median survival times for the low- and high-protein expression subgroups, respectively, are 202 days [95\% confidence interval (CI), 83 to 689] vs. 695 days (95\% CI, 465 to 1182) for growth hormone [hazard ratio (HR), 0.309; $\mathrm{p}=0.006$ ], 155 days (95\% CI, 57 to 689) vs. 684 days (95\% CI, 465 to 1182) for IgE (HR, 0.303; p=0.004), 482 days (95\% CI, 465 to 1182) vs. 188 days (95\% CI, 94 to 962) for IL-16 (HR, 2.49; p=0.025), and 695 days (95\% CI, 470 to 1182) vs. 276 days (95\% CI, 83 to 689) for VEGF (HR, 3.13; $\mathrm{p}=0.006$ ).

\section{Discussion}

This study demonstrated the feasibility of using multiplex immunoassay to identify novel and interesting blood-based protein biomarkers for malignant glioma. Both unique and common biomarker candidates associated with malignant glioma were found using plasma and serum samples. In addition, protein biomarker candidates associated with malignant glioma prognosis were identified.

Consistent with previous studies, we identified protein biomarkers known to be elevated in human malignant glioma specimens. For example, serum IL-10 level has been previously described as significantly increased in high-grade glioma patients compared with non-tumor control patients (13). Similarly, IL-10 was identified in our study as a serum biomarker that accurately classified GBM patients from controls. As a potent anti-inflammatory type II cytokine, increased levels of IL-10 in GBM patients is consistent with the findings that Th2 cytokines are elevated in patients with advanced tumors $(14,15)$. Plasma matrix metalloproteinases-2 (MMP-2) and VEGF were identified in our study as potential plasma biomarkers that accurately distinguished high-grade glioma patients from controls. Of note, MMP-2 and VEGF also have been investigated as diagnostic biomarkers for brain tumors using urine samples (16). MMP-2, an extracellular matrix metalloproteinase that promotes cell invasion, angiogenesis, and activation of growth factors, is involved in the development of human glioma microvessels and facilitates glioma cell invasion (17). Significantly decreased levels of MMP-2 have been observed in cisplatin-treated human glioblastoma cell lines (18). VEGF can promote tumorigenesis and angiogenesis of human glioblastoma stem cells, and VEGF expression is significantly increased in high-grade astrocytomas compared with low-grade astrocytomas (19). Various anti-VEGF therapies are in development, and an anti-VEGF monoclonal antibody, Avastin (bevacizumab), was recently approved for treatment of glioblastoma (20). 19-9, SCF, factor VII, and MDC) were found to have similar classification accuracy and fold changes in two independent comparisons. To our knowledge, the differential expression levels for these proteins in high-grade glioma patients' blood have not been previously reported. Further study of their functions and expressions in glioma tissue or cell lines will improve our knowledge of their roles as potential circulating biomarkers for high-grade glioma. A2M has been found to be synthesized and secreted by human glioma cells and 
up-regulated in migrating human glioma cells compared with non-migrating glioma cells (21). The A2M receptor, LRP, is overexpressed in human malignant astrocytomas, especially in GBM (22), and A2M can cross the blood-brain barrier in an LRP-dependent manner (23). CA 19-9 is known to be a circulating protein biomarker for gastrointestinal cancers (24). Although the expression and function of CA 19-9 in human glioma tissue are not well understood, increased CA 19-9 expression has been detected during tumorigenesis in a C6 rat glioma model, with this expression decreased after 8-Cl-cAMP treatment (25). SCF is a growth factor important for cell survival, proliferation, and differentiation of hematopoietic and cancer stem cells (26). SCF can stimulate human GBM cell line growth (27). Neuronal and glioma-derived SCF can induce angiogenesis within the brain (28). Factor VII is a central protein in the blood coagulation cascade, and thrombosis frequently found in GBM (29). Expression of tissue factor, the cell surface receptor for factor VII, correlates with histologic grade of human glioma malignancy and vascularity (30). In addition to accurately classification of malignant glioma patients from controls, factor VII was also found to have prognostic significance using serum samples. Among these five biomarker candidates, MDC is the only protein underexpressed in the plasma and serum samples from highgrade glioma patients. MDC, or CCL22, is a member of the C-C motif chemokine family, which regulates $\mathrm{TH} 2$-related immune responses (31). Of note, MDC and its receptor CCR4 both show decreased expression in human grade III astrocytoma and grade IV glioblastoma cells, compared with adult human astrocytes (32).

As a potent chemoattractant for lymphocytes, MIP-1 $\alpha$, or CCL3, plays important roles in inflammation, chemotaxis, and immune response (33). Although human astrocytoma cells are capable of producing MIP-1s (10), the expression of MIP-1 $\alpha$ at the protein level in different grades of human glioma tissues has not been well studied. Our study showed increased MIP-1 $\alpha$ protein expression levels in high-grade gliomas when compared with grade II astrocytomas. In addition, our study showed that MIP-1 $\alpha$ can directly stimulate glioblastoma cell proliferation and migration. These results are consistent with the previous findings that the mRNA of CC ligand 3-like protein 1 (CCL3L1), a member of the MIP-1 family, was overexpressed in glioblastoma compared to levels in lower-grade gliomas, and transfection of CCL3L1 enhanced the glioblastoma cell line proliferation (34).

Our study also identified potential prognostic biomarkers for high-grade glioma. Our findings for $\operatorname{IgE}$ is consistent with a previous report that elevated circulating $\operatorname{IgE}$ is associated with better survival in high-grade glioma patients (35). The correlation between circulating IL-16 and growth hormone with glioma patient survival were not well-known. At the tissue level, the percentage of IL-16 positive cells has been shown to be increased significantly in studies comparing control human brains and WHO grade II brain tumors to anaplastic astrocytoma and GBM (36). GH, a pituitary hormone, has been shown to enhance natural killer cell activity against glioma (37).

For future studies, because the number of high-grade glioma cases enrolled in this study was relatively small, a larger cohort of samples should be analyzed to further validate these protein biomarkers. Considering the low incidence rate of glioma, it is not likely that a general screening test will be useful. Comparison of blood samples from normal versus malignant glioma patients may have found biomarkers that are associated with changes to the blood-brain barrier, which are not specific to cancer. Differentiating patients with various brain pathologies from gliomas will be important. Measurement of serial protein levels during the course of malignant glioma development would also be likely to be more informative. In addition, bloodbased molecular biomarkers for monitoring high-grade glioma recurrence or assessing response to treatment should also be explored.

Overall, our proteomic approach identified a list of previously unknown blood-based protein biomarkers that may aid in clinical assessment of malignant glioma patients. We demonstrated that both plasma and serum are important blood samples and are complementary in biomarker discovery. Multiplex immunoassay of blood-based protein biomarkers is shown to be a promising tool for advancing personalized medicine for glioma patients.

\section{Acknowledgements}

The authors thank for Dr Yinghao Su for his assistance with the quantitative analysis of IHC, and Lynne Berry and Yvonne Poindexter from the VUMC Biostatistics Department for their comments regarding the manuscript. We also thank the Brain Tumor Foundation of Canada for sharing their GBM serum samples. This study was supported by The Brain Tumor Funders' Collaborative Grant and American Brain Tumor Association Translational Grant.

\section{References}

1. Gerstner ER and Fine RL: Increased permeability of the bloodbrain barrier to chemotherapy in metastatic brain tumors: establishing a treatment paradigm. J Clin Oncol 25: 2306-2312, 2007.

2. Quaranta M, Divella R, Daniele A, et al: Epidermal growth factor receptor serum levels and prognostic value in malignant gliomas. Tumori 93: 275-280, 2007.

3. Ilzecka J and Ilzecki M: APRIL is increased in serum of patients with brain glioblastoma multiforme. Eur Cytokine Netw 17: 276-280, 2006

4. Brommeland T, Rosengren L, Fridlund S, Hennig R and Isaksen V: Serum levels of glial fibrillary acidic protein correlate to tumour volume of high-grade gliomas. Acta Neurol Scand 116: 380-384, 2007.

5. Lin Y, Jiang T, Zhou K, et al: Plasma IGFBP-2 levels predict clinical outcomes of patients with high-grade gliomas. Neuro Oncol 11: 468-476, 2009.

6. Amonkar SD, Bertenshaw GP, Chen TH, et al: Development and preliminary evaluation of a multivariate index assay for ovarian cancer. PLoS One 4: e4599, 2009.

7. Rush SZ, Abel TW, Valadez JG, Pearson M and Cooper MK: Activation of the Hedgehog pathway in pilocytic astrocytomas. Neuro Oncol 12: 790-798, 2010.

8. Su Y, Shrubsole MJ, Ness RM, et al: Immunohistochemical expressions of Ki-67, cyclin D1, beta-catenin, cyclooxygenase-2, and epidermal growth factor receptor in human colorectal adenoma: a validation study of tissue microarrays. Cancer Epidemiol Biomarkers Prev 15: 1719-1726, 2006.

9. Ponten F, Jirstrom K and Uhlen M: The Human Protein Atlas - a tool for pathology. J Pathol 216: 387-393, 2008.

10. Ishii N, Tada M, Sakuma S, Sawamura Y, Shinohe Y and Abe H: Human astrocytoma cells are capable of producing macrophage inflammatory protein-1beta. J Neurooncol 37: 17-23, 1998.

11. Terpos E, Politou M, Viniou N and Rahemtulla A: Significance of macrophage inflammatory protein-1 alpha (MIP-1alpha) in multiple myeloma. Leuk Lymphoma 46: 1699-1707, 2005. 
12. Lentzsch S, Gries M, Janz M, Bargou R, Dorken B and Mapara MY: Macrophage inflammatory protein 1-alpha (MIP-1 alpha) triggers migration and signaling cascades mediating survival and proliferation in multiple myeloma (MM) cells. Blood 101: 3568-3573, 2003.

13. Kumar R, Kamdar D, Madden L, et al: Th1/Th2 cytokine imbalance in meningioma, anaplastic astrocytoma and glioblastoma multiforme patients. Oncol Rep 15: 1513-1516, 2006.

14. Dummer W, Becker JC, Schwaaf A, Leverkus M, Moll T and Brocker EB: Elevated serum levels of interleukin-10 in patients with metastatic malignant melanoma. Melanoma Res 5: 67-68, 1995.

15. Shibata M, Nezu T, Kanou H, Abe H, Takekawa $M$ and Fukuzawa M: Decreased production of interleukin-12 and type 2 immune responses are marked in cachectic patients with colorectal and gastric cancer. J Clin Gastroenterol 34: 416-420, 2002.

16. Smith ER, Zurakowski D, Saad A, Scott RM and Moses MA: Urinary biomarkers predict brain tumor presence and response to therapy. Clin Cancer Res 14: 2378-2386, 2008.

17. Girolamo F, Virgintino D, Errede M, et al: Involvement of metalloprotease- 2 in the development of human brain microvessels. Histochem Cell Biol 122: 261-270, 2004.

18. Chintala SK, Ali-Osman F, Mohanam S, et al: Effect of cisplatin and BCNU on MMP-2 levels in human glioblastoma cell lines in vitro. Clin Exp Metastasis 15: 361-367, 1997.

19. Hlobilkova A, Ehrmann J, Knizetova P, Krejci V, Kalita O and Kolar Z: Analysis of VEGF, Flt-1, Flk-1, nestin and MMP-9 in relation to astrocytoma pathogenesis and progression. Neoplasma 56: 284-290, 2009

20. Norden AD, Drappatz J and Wen PY: Antiangiogenic therapies for high-grade glioma. Nat Rev Neurol 5: 610-620, 2009.

21. Zagzag D, Salnikow K, Chiriboga L, et al: Downregulation of major histocompatibility complex antigens in invading glioma cells: stealth invasion of the brain. Lab Invest 85: 328-341, 2005.

22. Yamamoto $M$, Ikeda $K$, Ohshima $K$, Tsugu $H$, Kimura $H$ and Tomonaga M: Increased expression of low density lipoprotein receptor-related protein/alpha2-macroglobulin receptor in human malignant astrocytomas. Cancer Res 57: 2799-2805, 1997.

23. Demeule M, Currie JC, Bertrand Y, et al: Involvement of the lowdensity lipoprotein receptor-related protein in the transcytosis of the brain delivery vector angiopep-2. J Neurochem 106 1534-1544, 2008.

24. Duffy MJ: CA $19-9$ as a marker for gastrointestinal cancers: a review. Ann Clin Biochem 35: 364-370, 1998.
25. Andrijevic L: The influence of 8-Cl-cAMP on tumor marker CA19.9 on experimental glioma in rats. Arch Oncol 9: 9-12, 2001.

26. Ray P, Krishnamoorthy $\mathrm{N}$ and Ray A: Emerging functions of c-kit and its ligand stem cell factor in dendritic cells: regulators of T cell differentiation. Cell Cycle 7: 2826-2832, 2008.

27. Berdel WE, de Vos S, Maurer J, et al: Recombinant human stem cell factor stimulates growth of a human glioblastoma cell line expressing c-kit protooncogene. Cancer Res 52: 3498-3502, 1992.

28. Sun L, Hui AM, Su Q, et al: Neuronal and glioma-derived stem cell factor induces angiogenesis within the brain. Cancer Cell 9: 287-300, 2006

29. Tehrani M, Friedman TM, Olson JJ and Brat DJ: Intravascular thrombosis in central nervous system malignancies: a potential role in astrocytoma progression to glioblastoma. Brain Pathol 18: 164-171, 2008.

30. Guan M, Jin J, Su B, Liu WW and Lu Y: Tissue factor expression and angiogenesis in human glioma. Clin Biochem 35: 321-325, 2002.

31. Yamashita U and Kuroda E: Regulation of macrophage-derived chemokine (MDC, CCL22) production. Crit Rev Immunol 22: 105-114, 2002.

32. Maru SV, Holloway KA, Flynn G, et al: Chemokine production and chemokine receptor expression by human glioma cells: role of CXCL10 in tumour cell proliferation. J Neuroimmunol 199: 35-45, 2008.

33. Maurer M and von Stebut E: Macrophage inflammatory protein-1. Int J Biochem Cell Biol 36: 1882-1886, 2004.

34. Kouno J, Nagai H, Nagahata T, et al: Up-regulation of CC chemokine, CCL3L1, and receptors, CCR3, CCR5 in human glioblastoma that promotes cell growth. J Neurooncol 70: 301-307, 2004.

35. Wrensch M, Wiencke JK, Wiemels J, et al: Serum IgE, tumor epidermal growth factor receptor expression, and inherited polymorphisms associated with glioma survival. Cancer Res 66: 4531-4541, 2006.

36. Liebrich M, Guo LH, Schluesener HJ, et al: Expression of interleukin-16 by tumor-associated macrophages/activated microglia in high-grade astrocytic brain tumors. Arch Immunol Ther Exp (Warsz) 55: 41-47, 2007.

37. Shimizu K, Adachi K and Teramoto A: Growth hormone enhances natural killer cell activity against glioma. J Nippon Med Sch 72: 335-340, 2005. 